\title{
Martingales and Stochastic Analysis
}




\section{SERIES ON MULTIVARIATE ANALYSIS}

Editor: M M Rao

\section{Forthcoming}

Vol. 2: Mathematical Methods on Sample Surveys

H. F. Tucker 


\section{Mulitivariate}

\section{Analysis}

- Vol. 1-

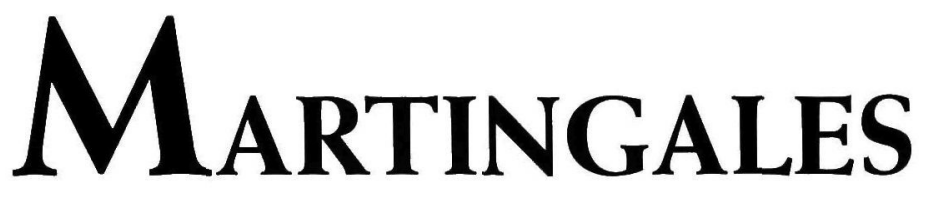

AND

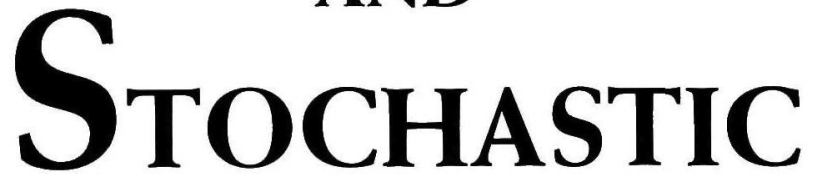

ANALYSIS

\section{J. Yeh}

Department of Matbematics

University of California, Irvine 


\section{Published by}

World Scientific Publishing Co. Pte. Ltd.

P O Box 128, Farrer Road, Singapore 912805

USA office: Suite 1B, 1060 Main Street, River Edge, NJ 07661

UK office: 57 Shelton Street, Covent Garden, London WC2H 9HE

\section{Library of Congress Cataloging-in-Publication Data \\ Yeh, James. \\ Martingales and stochastic analysis / James Yeh.}

p. $\quad \mathrm{cm}$. -- (Series on multivariate analysis ; vol. 1)

Includes bibliographical references and index.

ISBN $981022477 \mathrm{X}$

1. Martingales (Mathematics) 2. Stochastic analysis. I. Title.

II. Series.

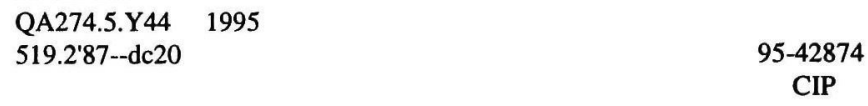

\section{British Library Cataloguing-in-Publication Data}

A catalogue record for this book is available from the British Library.

Copyright $(1995$ by World Scientific Publishing Co. Pte. Ltd.

All rights reserved. This book, or parts thereof, may not be reproduced in any form or by any means, electronic or mechanical, including photocopying, recording or any information storage and retrieval system now known or to be invented, without written permission from the Publisher.

For photocopying of material in this volume, please pay a copying fee through the Copyright Clearance Center, Inc., 222 Rosewood Drive, Danvers, MA01923, USA.

This book is printed on acid-free paper. 


\section{Preface}

This monograph is an introduction to martingales and stochastic analysis assuming only real analysis and some basic concepts in probability theory. Stochastic independence, conditional expectation, and regular conditional probability are included in the appendix. In writing this monograph I strove for clarity and precision at the cost of concision. Details of proofs are worked out for readability and for reference.

Chapter 1 begins with a collection of theorems concerning $\sigma$-algebras that will be need in the sequel. Measurable processes, progressively measurable processes, right-continuous and left-continuous processes, predictable processes, and approximation by sequences of simple processes are discussed Stopping times are introduced as a method of truncation for stochastic processes. Uniform integrability, which plays an important role in martingale theory, is included.

Chapter 2 introduces martingale and submartingale properties as monotonicity conditions in terms of conditional expectation. The chapter includes the fundamental martingale inequalities, optional stopping theorem, optional sampling theorem, martingale convergence theorem, closing a martingale by a final element, roles of uniform integrability in convergence questions for martingales, increasing processes as a class of submartingales, increasing processes as integrators in stochastic integration, and the Doob-Meyer decomposition theorem.

Chapter 3 treats Itô's stochastic integral in the language of martingale theory. The integrand is a left-continuous, and more generally a predictable, process satisfying some integrability condition on the sample functions and the integrator process is a right-continuous $L_{2}$-martingale. The stochastic integral is then extended to local $L_{2}$-martingales as integrators. The chapter also includes Itô's formula for quasimartingales and Itô's stochastic calculus.

Chapter 4 starts with a study of the space of continuous functions on which solutions of stochastic differential equations will be constructed. Function space representation of solutions, initial value problems, uniqueness of solution in the sense of probability law, pathwise uniqueness of solution, existence and uniqueness of strong solutions are the topics included in this chapter.

The main part of the text consists of four chapters in twenty sections: $\S 1$ to $\S 20$. One counter for all the twenty sections runs through the chapters. Within each section, one counter is used for definitions, lemmas, propositions, theorems, corollaries, remarks, observations, and examples. Thus for instance in $\S 5$, Definition 5.1 is followed by Definition 
5.2, Theorem 5.3, Proposition 5.4, Observation 5.5, and so on. Most sections are divided in to subsections and these are numbered with upper case roman numerals. For instance $\S 5$ is divided into five subsections: [I] to [V]. Numbering of definitions, lemmas, propositions, etc., in a section is independent of the subsections. The appendix has four parts: A to $\mathrm{D}$. Within each one of the four parts in the appendix, one counter is used for definitions, lemmas, propositions, etc. Thus for instance in Appendix A, Definition A.1 is followed by Proposition A.2, Observation A.3, Theorem A.4, and so on.

In writing this monograph I am indebted to [21] P. A. Meyer and [7] N. Ikeda and S. Watanabe as valuable guides. It is hoped that this monograph will also serve as an introduction as well as a supplement to the latter. I wish to express my gratitude to Dr. G. Engl and N. Malik for their comments on the manuscript. I thank Professor M. M. Rao for inviting me to write this monograph. Finally I express my appreciation to the editorial staff of World Scientific Publishing Co. for their cooperation in the production of the manuscript. 


\section{Contents}

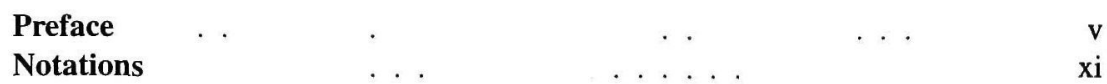

1 Stochastic Processes 1

$\S 1$ Generated $\sigma$-algebras . . . . . . . . . . . . . . $\quad 1$

$\S 2$ Stochastic Processes . . . . . . . . . . . . 11

[I] Measurable and Progressively Measurable Processes 11

[II] Predictable Processes $\quad$. . . . 19

$\S 3 \quad$ Stopping Times . 25

[I] Stopping Times for Stochastic Processes with Continuous Time 25

[II] Stopping Times for Stochastic Processes with Discrete Time . 39

[III] Random Variables at Stopping Times . 41

[IV] Stopped Processes and Truncated Processes . . 45

$\S 4$ Convergence in $L_{p}$ and Uniform Integrability . . . 49

[I] Convergence in $L_{p} \quad$. . . . . . . . . 49

[II] Uniformly Integrable Systems of Random Variables $\quad . \quad 53$

2 Martingales $\quad 71$

$\S 5$ Martingale, Submartingale and Supermartingale . $\quad 71$

[I] Martingale, Submartingale and Supermartingale Properties . 71

[II] Convexity Theorems _. $\quad \ldots$. . . .

[III] Discrete Time Increasing Processes and Doob Decomposition 78

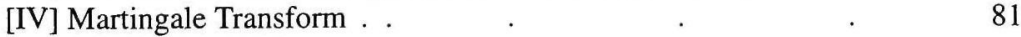

[V] Some Examples ～. . . . . $\quad$. $\quad$. 84

§6 Fundamental Submartingale Inequalities _ . . . . $\quad 86$

[I] Optional Stopping and Optional Sampling _ . . $\quad$. $\quad 87$

[II] Maximal and Minimal Inequalities . $\quad$. . $\quad$. $\quad 93$

[III] Upcrossing and Downcrossing Inequalities . 101 
$\S 7$ Convergence of Submartingales . . . . . . . . . . . . . . 110

[I] Convergence of Submartingales with Discrete Time . . . . . . 110

[II] Convergence of Submartingales with Continuous Time $\quad$. . . . . . 114

[III] Closing a Submartingale with a Final Element . . . . . . . . 116

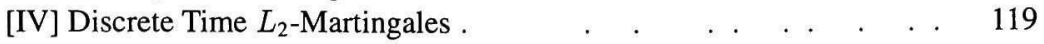

$\S 8$ Uniformly Integrable Submartingales . . . . . . . . . . . 122

[I] Convergence of Uniformly Integrable Submartingales . . . 122

[II] Submartingales with Reversed Time . . . . . . . . . 124

[III] Optional Sampling by Unbounded Stopping Times . . _ . . . 131

[IV] Uniform Integrability of Random Variables at Stopping Times . . 141

$\S 9$ Regularity of Sample Functions of Submartingales . . . . . . 145

[I] Sample Functions of Right-Continuous Submartingales . . . 145

[II] Right-Continuous Modification of a Submartingale . . 149

$\S 10$ Increasing Processes . . . . $\quad \ldots \quad \ldots 155$

[I] The Lebesgue Stieltjes Integral . . . . . $\quad$. . 155

[II] Integration with Respect to Increasing Processes . . . . . . 158

[III] Doob-Meyer Decomposition ～. . . . . . . . . 171

[IV] Regular Submartingales $\quad$. . . . . . . . 187

3 Stochastic Integrals $\quad 197$

$\S 11 \quad L_{2}$-Martingales and Quadratic Variation Processes . . . . . . . . . 197

[I] The Space of Right-Continuous $L_{2}$-Martingales . . . . . . . . . . . 197

[II] Signed Lebesgue-Stieltjes Measures _ . . . . . . . . . . 205

[III] Locally Bounded Variation Processes ～. . . . . . . 207

[IV] Quadratic Variation Processes . . . . . 212

$\S 12$ Stochastic Integrals with Respect to Martingales . . . . . . . . . . . . . 221

[I] Stochastic Integral of Bounded Left-Continuous Adapted

Simple Processes w. r. t. $L_{2}$-Martingales ‥ . . . . 222

[II] Stochastic Integral of Predictable Processes w. r. t. $L_{2}$-Martingales $\quad 235$

$\S 13$ Adapted Brownian Motions . . . $\quad$. . . . . $\quad$. . 262

[I] Processes with Independent Increments ～. . . . . . . . . . 262

[II] Brownian Motions in $\mathbb{R}^{d}$. . . . . . . . . . . 270

[III] 1-Dimensional Brownian Motions . $\quad \ldots \ldots \ldots \ldots$. . . 286

[IV] Stochastic Integrals with Respect to a Brownian Motion . . 290

$\S 14$ Extensions of the Stochastic Integral . . . . . 297

[I] Local $L_{2}$-Martingales and Their Quadratic Variation Processes _ . . 297

[II] Extensions of the Stochastic Integral to Local Martingales . . . . 306 


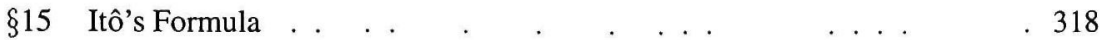

[I] Continuous Local Semimartingales and Itô's Formula . . 318

[II] Stochastic Integrals with Respect to Quasimartigales _ . . . 333

[III] Exponential Quasimartingales . . . . . . 335

[IV] Multidimensional Itô's Formula . . 340

§16 Itô's Stochastic Calculus . . . . 344

[I] The Space of Stochastic Differentials _. . 344

[II] Fisk-Stratonovich Integrals . . $\quad \ldots \quad . \quad \ldots 352$

4 Stochastic Differential Equations $\quad 357$

$\S 17$ The Space of Continuous Functions . . 357

[I] Function Space Representation of Continuous Processes _ . . 357

[II] Metrization of the Space of Continuous Functions $\quad .364$

$\S 18$ Definition and Function Space Representation of Solution 368

[I] Definition of Solutions ․ . . . . . . 368

[II] Function Space Representation of Solutions . . 372

[III] Initial Value Problems $\quad \ldots . \quad$. 385

$\S 19$ Existence and Uniqueness of Solutions . . . 395

[I] Uniqueness in Probability Law and Pathwise Uniqueness of Solutions 395

[II] Simultaneous Representation of Two Solutions on a Function Space . . 405

$\S 20$ Strong Solutions . . . . . . . . . . . . . . . . . 419

[I] Existence of Strong Solutions $\quad$. 419

[II] Uniqueness of Strong Solutions 432

A Stochastic Independence $\quad 443$

B Conditional Expectations 453

C Regular Conditional Probabilities $\quad 475$

D Multidimensional Normal Distributions $\quad 487$

Bibliography . . . . . . . . . . . 495

Index .. . . . . . . . . . . . . . . . 498 
This page is intentionally left blank 


\section{Notations}

$\mathbb{N}$

$\mathbb{Z}$

$\mathbb{Z}_{+}$

$\mathbb{R}$

$\mathbb{R}_{+}$

$\mathbb{T}$

$\overline{\mathbb{N}}$

$\overline{\mathbb{Z}}_{+}$

$\overline{\mathbb{R}}_{+}$

$\overline{\mathbb{T}}$

$\sigma(\mathfrak{E})$

$\alpha(\mathfrak{E})$

$d(\mathfrak{E})$

$\sigma(f)$

$\left(\Omega, \mathfrak{F},\left\{\mathfrak{F}_{t}\right\}, P\right)$

$X^{T \wedge}$

$X^{[T]}$

$P \lim _{n \rightarrow \infty} X_{n}$

$C \cdot X$

$\mathbf{M}_{2}\left(\Omega, \mathfrak{F},\left\{\mathfrak{F}_{t}\right\}, P\right)$

the natural numbers

the integers

the nonnegative integers

the real numbers

$[0, \infty)$

$\mathbb{R}_{+}$or $\mathbb{Z}_{+}$

$\mathbb{N} \cup\{\infty\}$

$\mathbb{Z}_{+} \cup\{\infty\}$

$\mathbb{R}_{+} \cup\{\infty\}$

$\mathbb{T} \cup\{\infty\}$

$\{-\infty\} \cup \mathbb{R} \cup\{\infty\}$

$\sigma$-algebra generated by $\mathfrak{E}$

algebra generated by $\mathfrak{E}$

$d$-class generated by $\mathfrak{E}$

$\sigma$-algebra generated by $f$

a filtered space

stopped process of $X$ by stopping time $T$

truncated process of $X$ by stopping time $T$

limit of convergence in probability measure $P$

martingale transform

space of null at 0 right-continuous $L_{2}$ martingales on a

standard filtered space

$\mathbf{M}_{2}^{c}\left(\Omega, \mathfrak{F},\left\{\mathfrak{F}_{t}\right\}, P\right)$

| $\left.\right|_{\infty} ^{t}$

$\mathbf{M}_{2}^{l o c}\left(\Omega, \mathfrak{F},\left\{\mathfrak{F}_{t}\right\}, P\right)$

space of null at 0 a. s. continuous $L_{2}$ martingales on a

standard filtered space

a seminorm on $\mathbf{M}_{2}\left(\Omega, \mathfrak{F},\left\{\mathfrak{F}_{t}\right\}, P\right)$

a quasinorm on $\mathbf{M}_{2}\left(\Omega, \mathfrak{F},\left\{\mathfrak{F}_{t}\right\}, P\right)$

198

198

space of null at 0 right-continuous local $L_{2}$ martingales on a standard filtered space

300

$\mathbf{M}_{2}^{\text {cloc }}\left(\Omega, \mathfrak{F},\left\{\mathfrak{F}_{t}\right\}, P\right)$ space of null at 0 a. s. continuous local $L_{2}$ martingales on a standard filtered space

300

[M]

quadratic variation process of $M$

214

quadratic variation process of $M$ and $N$ 
$\mathbf{A}\left(\Omega, \mathfrak{F},\left\{\mathfrak{F}_{t}\right\}, P\right) \quad$ space of a.s. increasing processes on a standard filtered space

$\mathbf{A}^{c}\left(\Omega, \mathfrak{F},\left\{\mathfrak{F}_{t}\right\}, P\right) \quad$ space of a.s. continuous a.s. increasing processes on a standard filtered space

$\mathbf{A}^{l o c}\left(\Omega, \mathfrak{F},\left\{\mathfrak{F}_{t}\right\}, P\right)$

see Definition 14.6

$\mathbf{V}\left(\Omega, \mathfrak{F},\left\{\mathfrak{F}_{t}\right\}, P\right)$

space of a.s. locally bounded variation processes on a standard filtered space

$\mathbf{V}^{c}\left(\Omega, \mathfrak{F},\left\{\mathfrak{F}_{t}\right\}, P\right)$

space of a.s. continuous a.s. locally bounded variation processes on a standard filtered space

$\mathbf{V}^{l o c}\left(\Omega, \mathfrak{F},\left\{\mathfrak{F}_{t}\right\}, P\right)$

see Definition 14.6

210

see Definition 14.6

total variation process of $V \in \mathbf{V}\left(\Omega, \mathfrak{F},\left\{\mathfrak{F}_{t}\right\}, P\right)$

300

$\mathbf{V}^{c, l o c}\left(\Omega, \mathfrak{F},\left\{\mathfrak{F}_{t}\right\}, P\right)$

\section{$|V|$}

$m_{L}$

$m_{W}$

$\mu_{A}$

$\int_{[0, t]} X(s) d A(s)$

$\mathbf{L}_{p, \infty}\left(\mathbb{R}_{+} \times \Omega, \mu_{A}, P\right)$

$\|\cdot\|_{P, P}^{A, P}$

$\|\cdot\|_{p, \infty}^{A, P}$

$\mathbf{L}_{2, \infty}^{\text {loc }}\left(\mathbb{R}_{+} \times \Omega, \mu_{A}, P\right)$

$\mathbf{L}_{1, \infty}^{\text {loc }}\left(\mathbb{R}_{+} \times \Omega, \mu_{A}, P\right)$

$\mathbf{L}_{p, \infty}\left(\mathbb{R}_{+} \times \Omega, m_{L} \times P\right)$

$\|\cdot\|_{p, t}^{m_{L} \times P}$

$\|\cdot\|_{p, \infty}^{m_{L} \times P}$

$\mathbf{L}_{0}\left(\Omega, \mathfrak{F},\left\{\mathfrak{F}_{t}\right\}, P\right)$

$X \bullet M$

$\int_{[0, t]} X(s) d M(s)$ the Lebesgue measure

the Wiener measure

family of Lebesgue-Stieltjes measures determined by an a.s. increasing process $A$

alternate for $\int_{[0, t]} X(s) \mu_{A}(d s)$

see Definition 11.17

a seminorm on $\mathbf{L}_{p, \infty}\left(\mathbb{R}_{+} \times \Omega, \mu_{A}, P\right)$

a quasinorm on $\mathbf{L}_{p, \infty}\left(\mathbb{R}_{+} \times \Omega, \mu_{A}, P\right)$

see Definition 14.14

see Definition 15.8

see Definition 13.35

a seminorm on $\mathbf{L}_{p, \infty}\left(\mathbb{R}_{+} \times \Omega, m_{L} \times P\right)$

a quasinorm on $\mathbf{L}_{p, \infty}\left(\mathbb{R}_{+} \times \Omega, m_{L} \times P\right)$

the collection of all bounded adapted left-continuous processes on a standard filtered space $\left(\Omega, \mathfrak{F},\left\{\mathfrak{F}_{t}\right\}, P\right)$ stochastic integral of $X$ with respect to $M$

alternate for $(X \bullet M)_{t}$
300

208

59

362

159

212

212

212

307

333

292

292

292

222

223,313

223,314 
$\mathbf{C}\left(\mathbb{R}_{+} \times \Omega\right) \quad$ space of measurable processes with

$\mathbf{B}\left(\mathbb{R}_{+} \times \Omega\right)$

a.s. continuous sample functions

space of measurable processes with

a.s. locally bounded sample functions

$\mathbf{Q}\left(\Omega, \mathfrak{F},\left\{\mathfrak{F}_{t}\right\}, P\right)$

collection of all quasimartingales on a

standard filtered space

dQ

collection of the equivalence classes in

$\mathbf{Q}\left(\Omega, \mathfrak{F},\left\{\mathfrak{F}_{t}\right\}, P\right)$

$\mathbf{d M}_{2}^{c, l o c}, \mathbf{d V}^{c, l o c}$

subcollections of $\mathbf{d Q}$

$\mathbf{W}^{d}$

space of $d$-dimensional continuous functions

on $\mathbb{R}_{+}$

$\left(\mathbf{W}^{d}, \mathfrak{W}^{d}, m_{W}^{d}\right)$

d-dimensional Wiener space

362

$\left(\mathbf{W}^{d}, \mathfrak{W}^{d},\left\{\mathfrak{W}_{t}^{d}\right\}\right)$

see Definition 17.3

359

$\left(\mathbf{W}^{r}, \mathfrak{W}^{r, w},\left\{\mathfrak{W}_{t}^{r, w}\right\}, m_{W}^{r}\right)$

see Definition 19.10

406

$\mathbf{M}^{d \times r}\left(\mathbf{W}^{d}, \mathfrak{W}^{d},\left\{\mathfrak{W}_{t}^{d}\right\}\right)$

see Definition 18.2

369

$\left(\mathbf{W}^{r+d}, \mathfrak{W}^{r+d, *},\left\{\mathfrak{W}_{t}^{r+d, *}\right\}, P_{(B, X)}\right)$

see Definition 18.10

374

$\left(\mathbf{W}^{r+d}, \mathfrak{W}^{r+d, *, x},\left\{\mathfrak{W}_{t}^{r+d, *, x}\right\}, P_{(B, X)}^{x}\right)$

see Definition 18.17

387

$\mathbf{L}_{2, \infty}^{d, c}\left(\mathbb{R}_{+} \times \Omega,\left\{\mathfrak{F}_{t}\right\}, m_{L} \times P\right)$

see Definition 20.2

419 